\title{
Acessibilidade ao cuidado na Estratégia de Saúde da Família no Oeste Baiano
}

\author{
Family Health Strategy Care Accessibility in West Bahia
}

Milena Marques Mendonça (https://orcid.org/0000-0003-3029-0240) ${ }^{1}$

Ítalo Ricardo Santos Aleluia (https://orcid.org/0000-0001-9499-6360) ${ }^{1}$

Maria Lidiany Tributino de Sousa (https://orcid.org/0000-0002-2332-8821) ${ }^{1}$

Marcos Pereira (https://orcid.org/0000-0002-2432-0653) ${ }^{2}$
${ }^{1}$ Universidade Federal do Oeste da Bahia. R. Professor José Seabra de Lemos 306, Recanto dos Pássaros. 47808-021 Barreiras BA Brasil.milenamarquesm@ hotmail.com

${ }^{2}$ Instituto de Saúde Coletiva, Universidade Federal da Bahia. Salvador BA Brasil.

\begin{abstract}
This study assessed the Family Health Strategy (ESF) health care accessibility in the municipal system, which is the health macro-region headquarters in Bahia. It consisted of two levels of analysis: the municipal management and the local organization of ESF teams. Data production combined documentary analysis, non-participant observation, and interviews with managers, professionals, and users. Goal-image was used with evaluative criteria and dimensions of the accessibility in Primary Care. Family Health Teams (EqSF) still do not entirely fulfill the role of preferential contact in municipal health services, and the health care accessibility reflects the interdependence of municipal and local factors. Rural and peripheral teams performed better in organizational accessibility, and central urban teams performed better in geographic accessibility. The assessment focused on geographic and organizational criteria, combining different sources of evidence and health system players using analysis levels considering the municipal and local PHC management are relevant contributions of this study, which can be extended to other municipal systems with similar characteristics.

Key words Health Services Accessibility, Primary Health Care, Family Health Strategy
\end{abstract}

Resumo Esse estudo avaliou a acessibilidade ao cuidado na Estratégia de Saúde da Família (ESF) em sistema municipal, sede de macrorregião de saúde no estado da Bahia. Tratou-se de estudo avaliativo em dois niveis de análise: a gestão $m u$ nicipal e a organização local das equipes da ESF. A produção dos dados combinou análise documental, observação não participante e entrevistas com gestores, profissionais e usuários. Utilizou-se imagem-objetivo com critérios e dimensões avaliativas da acessibilidade na Atenção Primária. As Equipes de Saúde da Família (EqSF) ainda não cumprem plenamente a função de contato preferencial nos sistemas municipais de saúde e a acessibilidade ao cuidado reflete interdependência de fatores municipais e locais. Equipes rurais e periféricas tiveram melhor desempenho na acessibilidade organizacional e equipes urbanas centrais melhor desempenho na acessibilidade geográfica. A avaliação centrada em critérios geográficos $e$ organizacionais, na combinação de diferentes fontes de evidência e atores do sistema de saúde, além do uso de niveis de análise considerando a gestão municipal e local da APS, são contribuições relevantes desse estudo, que podem ser ampliadas para outros sistemas municipais com características semelhantes.

Palavras-chave Acesso aos Serviços de Saúde, Atenção Primária à Saúde, Estratégia Saúde da Família 


\section{Introdução}

A Atenção Primária à Saúde (APS) corresponde a serviços de primeiro contato do usuário com o sistema de saúde, direcionados a resolver a maioria dos problemas de uma população ${ }^{1}$. No cenário internacional e nacional, a APS se destaca pelo papel principal de superar modelos de atenção fragmentados através do atributo da acessibilidade ${ }^{2}$.

No Brasil, a Estratégia de Saúde da Família (ESF) foi adotada como principal alternativa de reorganização do modelo assistencial e porta de entrada do Sistema Único de Saúde (SUS), através da ampliação da cobertura populacional dos serviços primários, desde a década de 1990. Esse movimento atribuiu ao poder municipal, responsabilidade político-administrativa para implantar e gerenciar Equipes de Saúde da Família $(\mathrm{EqSF})^{3}$.

O crescimento da expectativa de vida, as desigualdades regionais no acesso a serviços de saúde, o aumento da prevalência de condições crônicas, a manutenção de problemas de saúde emergentes e as causas externas de morbimortalidade geram importantes desafios para a ESF ordenar o processo de cuidado ${ }^{4}$. Esse cenário tem sido caracterizado por diversos autores como polarização epidemiológica que, diante de novos problemas emergentes como a pandemia de COVID-19 e contextos de grande desigualdade regional como o Nordeste brasileiro, aprofundam dificuldades das EqSF organizarem, gerirem e prestarem um cuidado ordenado.

Nesse sentido, a avaliação em saúde torna-se fundamental, uma vez que possibilita compreender sobre uma intervenção ou seus componentes, para apoiar a tomada de decisão em saúde ${ }^{5}$. A avaliação da acessibilidade na APS tem múltiplas conotações e, para esse estudo, consideraram-se características da oferta e o modo como as pessoas percebem e usam o serviço, a partir de elementos político-geográfico-organizacionais ${ }^{6}$.

Predominam mais desafios do que resultados positivos sobre o desempenho da APS no atributo da acessibilidade em sistemas de saúde. Resultados satisfatórios foram identificados, no Reino Unido e Estados Unidos (EUA), pela ampliação dos horários de atendimento no turno noturno, finais de semana e prazo estabelecido de 24 horas para atendimento 7 . No cenário nacional, melhores desempenhos relacionaram-se com a implantação de práticas de acolhimento, de escuta qualificada e de comunicação entre usuários e profissionais ${ }^{8}$.
Em países como a Austrália, Eslovênia e Escócia, a ausência de meios de transporte nos territórios limitava a escolha do horário de atendimento dos usuários na área rural ${ }^{9}$. Ainda no âmbito internacional, as desigualdades regionais de acesso geográfico eram mais expressivas em áreas remotas e rurais, com longo tempo de espera para marcação de consultas e atendimento e problemas com linhas telefônicas da APS prejudicavam a comunicação entre usuários e recepcionistas $^{10,11}$.

No Brasil, os desafios da acessibilidade na APS abarcam aspectos geográficos e organizacionais, simultaneamente. Os primeiros incluem a localização distante entre as unidades de saúde e residências, a infraestrutura local inadequada, a indisponibilidade de transporte, as demarcações territoriais equivocadas e a violência urbana ${ }^{7,9,12}$. Em relação aos aspectos organizacionais, há longo tempo de espera para atendimento, preferência dos usuários na busca por serviços de média e alta complexidade ${ }^{12}$, predominância de práticas centradas na doença ${ }^{13}$, utilização incompleta dos serviços primários, barreiras arquitetônicas para pessoas com deficiência, ações de acolhimento não implantadas ${ }^{14}$, descontinuidade do cuidado pela insuficiência de pessoal ${ }^{15}$, horários de atendimento pouco flexíveis, longo tempo de espera para realização de exames e de visita dos Agentes Comunitários de Saúde (ACS) ${ }^{16}$.

Avaliar a acessibilidade com o uso de diferentes abordagens metodológicas é relevante para qualificação dos serviços de saúde, e a análise da literatura internacional e nacional registra cenários heterogêneos na avaliação da acessibilidade. O enfoque dessas investigações na APS era predominantemente organizacional ${ }^{8-11,16}$. As abordagens metodológicas internacionais estavam centradas em estudos de abrangência nacional ${ }^{9,10}$ e as brasileiras em pesquisas de abrangência municipal em territórios urbanos metropolitanos ou capitais ${ }^{14-16}$. Ainda que nacionalmente predominassem estudos de enfoque municipal, pouco se identificou estudos avaliativos mistos, com ênfase para critérios geográficos e organizacionais, e conduzidos na APS de regiões de saúde remotas e com grande extensão territorial, como o Oeste baiano e o Nordeste do país. Por fim, grande parte dos estudos que avaliaram a acessibilidade na ESF não incluiu como nível de análise, a gestão municipal, limitando-se a evidências a partir da ótica de atores locais como usuários e profissionais de saúde 2-4,7-16. $^{2}$.

Este estudo buscou ampliar o espectro avaliativo considerando, também, a organização da 
gestão municipal, enquanto nível analítico capaz de elucidar relações contextuais entre as decisões políticas municipais e o desempenho local da acessibilidade nas EqSF. Nesta perspectiva, avaliou-se a acessibilidade ao cuidado na ESF em sistema municipal, sede da macrorregião de saúde no estado da Bahia.

\section{Metodologia}

\section{Delineamento e cenário do estudo}

Estudo avaliativo em dois níveis de análise: organização do sistema municipal de saúde e organização local das EqSF. O cenário do estudo foi em município de referência para 35 sistemas municipais de pequeno e médio porte, sendo o território mais populoso da macrorregião de saúde Oeste da Bahia e, com importante extensão territorial ${ }^{17}$.

A APS municipal encontrava-se em estágio de expansão com 29 EqSF implantadas e 63\% de cobertura populacional, dois Núcleos Ampliados de Saúde da Família e Atenção Básica (NASF-AB) tipo I e 12 Unidades Básicas de Saúde (UBS), sendo quatro de zona rural e oito de zona urbana.

\section{Critérios de seleção e coleta de dados}

$\mathrm{Na}$ análise do âmbito local das EqSF, selecionou-se quatro cenários avaliativos. Consideraram-se critérios que priorizaram a diversidade de características locais, a saber: localização, existência de estrutura física compartilhada com outra equipe, número de usuários adscritos, desempenho geral em estrutura e processo de trabalho, número de equipamentos sociais no território de cobertura, existência de apoio do NASF-AB, bem como de médico, enfermeiro e dentista com formação em Saúde da Família e Comunidade (Quadro 1).

Utilizou-se a análise documental dos Planos Municipais de Saúde (PMS) (2014-2017 e 20182021), dos Relatórios Anuais de Gestão (RAG), das Programações Anuais de Saúde (PAS) (20142018) e das agendas de trabalho das EqSF. Nesses documentos, buscou-se compreender em que medida a gestão municipal conseguiu estabelecer coerência entre as propostas dos PMS e ações implementadas e relativas aos critérios de avaliação da acessibilidade deste estudo.

Realizou-se entrevistas com profissionais, usuários e gestão municipal em 2018. Para seleção dos sujeitos utilizou-se o método de cadeia de informantes-chave, em especial, os Agentes Comunitários de Saúde (ACS) e gerentes das EqSF.

Participaram do estudo usuários com idade $\geq 60$ anos, cadastrados e adscritos às equipes, que utilizaram o serviço de APS nos últimos 15 dias e com capacidade de comunicação preservada. Os idosos foram priorizados como fonte de evidência, dada sua ampla utilização nas pesquisas de avaliação da acessibilidade. Os profissionais tinham, pelo menos, um ano de inserção nas EqSF e eram aqueles com maior envolvimento no processo de trabalho. Já os gestores, possuíam, pelo menos, um ano de atuação e eram lotados em atribuiçõos estratégicas para gestão das EqSF. Foram entrevistados 25 atores. Desses, 16 usuários, quatro enfermeiros, quatro médicos, o secretário municipal de saúde e o coordenador de APS.

\section{Análise de dados}

As informações produzidas foram trianguladas em processamento no software QRSNvivo 11, considerando os níveis de análise do estudo e critérios de avaliação da acessibilidade, propostos em imagem-objetivo por Cunha e Silva ${ }^{15}$, de domínio público e validada em estudo de caso municipal. A escolha metodológica da imagem -objetivo considerou sua amplitude analítica, por contemplar critérios tanto da gestão municipal quanto da organização local das EqSF, incluindo aspectos de acessibilidade geográfica - referentes à distância até o serviço e tempo de locomoção e organizacional - relativos ao tempo de espera, filas, sistemas de marcação de consultas e exames, horário de funcionamento, referência e contrarreferência, entre outros.

\section{Aspectos éticos}

O estudo foi aprovado em Comitê de Ética em Pesquisa em Seres Humanos. Entrevistados assinaram o termo de consentimento livre e esclarecido, com anuência em participar da pesquisa.

\section{Resultados}

\section{Organização do sistema}

O nível da organização da gestão municipal obteve pontuação satisfatória (18.5 do total de 25), ainda que entraves tenham sido identificados na garantia da APS como porta de entrada preferencial do sistema de saúde (Tabela 1). 
Quadro 1. Características dos cenários avaliativos do nível local das EqSF.

\begin{tabular}{|c|c|c|c|c|}
\hline \multirow{2}{*}{ Características } & \multicolumn{4}{|c|}{ Equipes } \\
\hline & A & B & $\mathrm{C}$ & D \\
\hline Localização & Rural & Urbana central & Urbana central & Urbana periférica \\
\hline Estrutura física compartilhada & Não & Não & Sim & Não \\
\hline Número de usuários adscritos & 292 & 4.708 & 1.520 & 4.126 \\
\hline $\begin{array}{l}\text { Desempenho geral em estrutura e } \\
\text { processo }\end{array}$ & Baixo & Baixo & Adequado & Adequado \\
\hline Número de equipamentos sociais & 2 & 3 & 6 & 1 \\
\hline Médico com residência em MFC & Não & Não & Não & Sim \\
\hline Enfermeiro com residência em SFC SF* $^{\star *}$ & Não & Não & Não & Sim \\
\hline Dentista com residência em SFC ${ }^{\star \star}$ & Não & Não & Não & Sim \\
\hline Dispõe de apoio do NASF-AB & Não & Não & Sim & Sim \\
\hline
\end{tabular}

*Medicina de Família e Comunidade; ${ }^{\star \star}$ Saúde da Família e Comunidade.

Fonte: elaboração dos autores.

Tabela 1. Pontuação da acessibilidade no nível de organização da gestão municipal.

\begin{tabular}{llcc}
\hline Dimensão & Critérios & $\begin{array}{c}\text { Gestão } \\
\text { Municipal }\end{array}$ & $\begin{array}{c}\text { Pontuação } \\
\text { máxima }\end{array}$ \\
\hline $\begin{array}{l}\text { Organização } \\
\text { do Sistema }\end{array}$ & $\begin{array}{l}\text { 1. Garantia } \\
\text { de acesso } \\
\text { como diretriz } \\
\text { de governo }\end{array}$ & 2,5 & 5 \\
& $\begin{array}{l}\text { 2. Rede } \\
\text { básica: porta } \\
\text { de entrada } \\
\text { aos demais } \\
\text { níveis }\end{array}$ & 16 & 20 \\
Pontuação & & 18,5 & \\
total & & & \\
\hline
\end{tabular}

Nota: classificação satisfatória - pontuação alcançada maior ou igual a 18 pontos, insatisfatória - menor ou igual a 17,5.

Fonte: elaboração dos autores.

A gestão municipal definiu propostas relacionadas com a implantação da ESF no PMS. Das 32 propostas identificadas, poucas delas (10) coadunavam com a garantia da acessibilidade como diretriz de governo. A minoria apresentava indícios claros de implantação nos Relatórios Anuais de Gestão (RAG). A análise dos RAG revelou persistência de cenário de contradição entre as propostas de governo municipal e as ações implantadas para garantia da acessibilidade na ESF. Isso foi observado pelo baixo número de ações cumpridas totalmente em relação às metas estabelecidas nas PAS.

Apesar da existência de diretrizes municipais relativas à garantia da acessibilidade na ESF, pre- dominou a priorização de aspectos da gestão e acessibilidade organizacional das equipes, com raras propostas relativas à dimensão geográfica da acessibilidade ao cuidado.

As propostas de governo municipal ainda permanecem circunscritas à ampliação de cobertura populacional da APS e pouco centradas na qualificação do processo de trabalho, externando tendência decisória para questões estruturais em detrimento dos fatores organizacionais, conforme ratificado nos PMS e RAG.

Os fatores relacionados com a organização do sistema municipal produziram constrangimentos gerenciais na prestação do cuidado local, com implicações diretas na garantia da acessibilidade. Critérios dependentes de decisões de governo municipal e atores externos ao território local extrapolavam a governabilidade das EqSF e interferiam no desempenho da acessibilidade.

A falta de uma diretriz municipal clara produzia cenários diversificados de organização do processo de marcação das consultas de APS. Embora esse cenário sugerisse certa autonomia da gerência local para definir o processo de marcação de consultas, criava barreiras de acessibilidade em equipes com agendas pouco flexíveis (apenas um dia da semana) ou para grupos de vulnerabilidade (idosos, gestantes, etc.).

Os documentos analisados e entrevistas com usuários e profissionais revelaram vazios de diretrizes municipais para instituir inovações nos processos de marcação de consultas nas EqSF, a exemplo da inexistência de marcações de consultas por telefone. Observou-se manutenção de modelo de organização de sistemas municipais, cujas vagas de consultas e exames especializados 
são distribuídos para as EqSF em regime de cotas, segundo critérios administrativos, desconsiderando as necessidades e características dos territórios. Os profissionais e gerentes foram unânimes em afirmar que essa lógica não atendia às necessidades das equipes, com longo tempo de espera entre a marcação e o acesso.

$\mathrm{Na}$ análise dos PMS registou-se proposições municipais para implantação de práticas de acolhimento e fortalecimento do vínculo da população com as EqSF, mas pouco se traduziram em ações concretas de Educação Permanente em Saúde para os profissionais.

Apesar da identificação de propostas municipais para estruturação do sistema de referência e contrarreferência nos PMS, a comunicação entre EqSF e demais pontos de atenção ainda é nodal para garantia da acessibilidade ao cuidado. Os profissionais indicaram a inoperância desse processo nas diversas situações, principalmente, da contrarreferência que raramente acontecia.

As unidades de saúde foram implantadas em locais improvisados e locados, demarcados em função da disponibilidade dos imóveis privativos e não mediante análise sociosanitária do território local. A localização distante entre a maioria das EqSF e o Centro de Atenção Especializada (CAE) e os serviços laboratoriais da rede própria era fator restritivo para a APS ordenar o cuidado para a AE, representando desigualdades territoriais importantes na acessibilidade geográfica.

\section{Organização local}

As EqSF ainda não cumpriam plenamente $\mathrm{o}$ atributo de acessibilidade. $\mathrm{O}$ desempenho em equipes pertencentes ao mesmo sistema municipal de saúde foi heterogêneo e classificado como intermediário, cuja variação foi $36,5-46,5$ pontos (Tabela 2). Profissionais e gestão afirmaram que constrangimentos para garantia da acessibilidade no âmbito local das EqSF estão relacionados com demanda de usuários no modelo queixa-conduta, para viabilizar o acesso a exames especializados; horários poucos flexíveis das unidades de saúde; barreiras geográficas de acesso dos usuários à atenção especializada e aos laboratórios; mau funcionamento do sistema de contrarreferência; e mecanismos polarizados de marcação de consultas por telefone.

Equipes em territórios rurais e periféricos tiveram melhor desempenho nos critérios da acessibilidade organizacional, em especial: gerenciamento da fila de espera, processo de acolhimento dos usuários; tempo de espera para marcação de consultas nas unidades de saúde; tempo de espera entre a marcação e o atendimento; e tempo de espera para ser atendido na consulta. Nas as equipes com características urbanas centrais melhores desempenhos envolveram critérios relativos à acessibilidade geográfica (Tabela 2).

O modelo de oferta de ações programáticas ainda domina as práticas de saúde em detrimento do modelo da oferta organizada. De acordo com as entrevistas de profissionais e gestores, a garantia da acessibilidade se dava mediante ações dos programas especiais de saúde.

Os principais indícios de reconhecimento, pelos usuários, das EqSF como serviço de primeiro acesso, foram relacionados ao conhecimento dos profissionais e da localização da unidade de saúde. As agendas de trabalho e entrevistas com profissionais e usuários ratificaram a ausência de inovações nos horários de funcionamento das unidades de saúde (predomina o horário administrativo) e que pouco contribuía para as EqSF serem porta de entrada preferencial do cuidado à noite e aos finais de semana.

Nas equipes rurais e periféricas, os horários de funcionamento das unidades de saúde foram mais restritos por questões de deslocamentos dos profissionais e de segurança, respectivamente. Em equipes urbanas centrais as restrições se relacionaram com descumprimento da carga horária, pelos profissionais, confirmada por usuários que relataram discrepâncias entre o horário definido pela gestão e o praticado pelas equipes.

Os obstáculos à acessibilidade na marcação e utilização de consultas de APS eram mais presentes em EqSF que adotavam agendamentos por grupos de vulnerabilidade, por dias ou turnos da semana, por senhas com número limitado de consultas diárias ou segregação de vagas entre demanda espontânea e organizada.

Os usuários, principalmente aqueles que residem distantes das unidades ou apresentavam limitações funcionais para deslocamentos, citaram os ACS como intermediadores na marcação de consultas.

As EqSF não tinham governabilidade para ordenar o cuidado para atenção especializada. Embora o processo de marcação de consultas e exames fosse descentralizado nas unidades de saúde, a distribuição da oferta entre os territórios adscritos era de domínio da Central de Regulação $\mathrm{Mu}$ nicipal, sem qualquer participação dos profissionais da APS. Territórios com características rurais e periféricas possuíam mais dificuldades de ordenar o acesso para a AE, pela ausência de marcadores para gerenciar agendamentos das consultas 
Tabela 2. Pontuação da acessibilidade ao cuidado no nível da organização local das EqSF.

\begin{tabular}{|c|c|c|c|c|c|c|}
\hline \multirow{2}{*}{ Dimensão } & \multirow{2}{*}{ Critérios } & \multicolumn{4}{|c|}{ Equipes } & \multirow{2}{*}{$\begin{array}{c}\text { Pontuação } \\
\text { máxima }\end{array}$} \\
\hline & & EqSF A & EqSF B & EqSF C & EqSF D & \\
\hline \multirow[t]{8}{*}{$\begin{array}{l}\text { Acessibilidade } \\
\text { organizacional }\end{array}$} & $\begin{array}{l}\text { 1. Horário de funcionamento das } \\
\text { unidades }\end{array}$ & 2,5 & 2,5 & 2,5 & 2,5 & 5 \\
\hline & 2. Sistema de marcação de consultas & 5 & 5 & 2,5 & 5 & 5 \\
\hline & 3. Marcação de consulta por telefone & 0 & 0 & 0 & 0 & 5 \\
\hline & $\begin{array}{l}\text { 4. Marcação de consultas e exames } \\
\text { especializados }\end{array}$ & 2,5 & 5 & 2,5 & 5 & 5 \\
\hline & 5. Lista de espera & 4 & 1,5 & 1,5 & 4 & 5 \\
\hline & 6. Acolhimento aos usuários & 4,5 & 4,5 & 3 & 3 & 5 \\
\hline & $\begin{array}{l}\text { 7. Encaminha através do sistema de } \\
\text { referência }\end{array}$ & 2,5 & 0 & 0 & 0 & 5 \\
\hline & 8. Recebe a contrarreferência & 0 & 0 & 0 & 0 & 5 \\
\hline Subtotal & & 21 & 18,5 & 12 & 19,5 & 40 \\
\hline \multirow[t]{4}{*}{$\begin{array}{l}\text { Barreiras } \\
\text { organizacionais }\end{array}$} & $\begin{array}{l}\text { 1. Tempo de espera para marcar } \\
\text { consulta }\end{array}$ & 5 & 0 & 0 & 5 & 5 \\
\hline & $\begin{array}{l}\text { 2. Tempo de espera entre a marcação e } \\
\text { o atendimento }\end{array}$ & 4,5 & 4 & 2,5 & 4,5 & 5 \\
\hline & $\begin{array}{l}\text { 3. Tempo de espera para ser atendido } \\
\text { na realização da consulta }\end{array}$ & 5 & 2,5 & 2,5 & 5 & 5 \\
\hline & 4. Filas para marcação de consultas & 5 & 2,5 & 0 & 5 & \\
\hline Subtotal & & 19,5 & 9 & 5 & 19,5 & 20 \\
\hline \multirow[t]{3}{*}{$\begin{array}{l}\text { Acessibilidade } \\
\text { Geográfica }\end{array}$} & $\begin{array}{l}\text { 1. Distância da residência do usuário } \\
\text { para a USF/UBS }\end{array}$ & 2 & 4,5 & 5 & 4 & 5 \\
\hline & $\begin{array}{l}\text { 2. Distância da residência para o CAE } \\
\text { e laboratório }\end{array}$ & 0 & 0 & 0 & 0 & 5 \\
\hline & 3. Existência de transporte & 3 & 4,5 & 4 & 3,5 & 5 \\
\hline Subtotal & & 5 & 9 & 9 & 7,5 & 15 \\
\hline Pontuação Total & & 45,5 & 36,5 & 26 & 46,5 & \\
\hline
\end{tabular}

Nota: classificação satisfatória - pontuação alcançada total maior que 54 pontos, intermediária - pontuação total maior que 27 e menor ou igual a 54 pontos, insatisfatória - pontuação total menor ou igual a 27 pontos.

Fonte: elaboração dos autores.

Tabela 3. Classificação geral da acessibilidade ao cuidado no cenário avaliativo, segundo os níveis municipal e local das Equipes de Saúde da Família. Município do Oeste da Bahia, 2018.

\begin{tabular}{lccccc}
\hline & EqSF A & EqSF B & EqSF C & EqSF D & $\begin{array}{c}\text { Pontuação } \\
\text { Máxima }\end{array}$ \\
\hline Total de pontos do município & 18,5 & 18,5 & 18,5 & 18,5 & 25 \\
Total de pontos das EqSF & 45,5 & 36,5 & 26 & 46,5 & 75 \\
Total & 64 & 55 & 45,5 & 65 & 100 \\
Classificação geral & Intermediária & Intermediária & Intermediária & Intermediária & - \\
\hline
\end{tabular}

Fonte: elaboração dos autores.

e exames, demonstrando que territórios remotos apresentam mais obstáculos nesse processo.

Apesar da maioria dos cenários avaliativos locais não se caracterizarem pela necessidade de transporte para deslocamento até as unidades de saúde, todos eles eram distantes geograficamente dos laboratórios e centros especializados, havendo, portanto, necessidade do uso de transporte público ou privado para acessar a atenção especializada. 
As entrevistas com profissionais registraram a recepção e desenvolvimento de salas de espera como principais espaços de acolhimentos dos usuários, distantes de estabelecer um processo de acolhimento transversal a outras práticas de saúde na ESF.

EqSF que funcionavam em espaço físico compartilhado com outras equipes apresentaram maior demanda reprimida, seja para marcação de procedimentos ou para atendimento. Esse modelo de organização estrutural favorecia aglomerações de pessoas, com longas filas que se iniciavam na madrugada.

Em síntese, as determinações municipais e locais foram responsáveis por produzir cenários polarizados no desempenho da acessibilidade ao cuidado em contextos com perfis e configurações distintas, não estando esse atributo, portanto, desvinculado de uma visão analítica sistêmica (Tabela 3).

\section{Discussão}

Diferenças de desempenho entre o nível da gestão municipal e local revelaram que a acessibilidade ao cuidado na ESF varia de acordo com as diretrizes municipais que orientam a organização da APS nos sistemas de saúde e da maneira como as EqSF organizam seu processo de trabalho junto ao território, ocorrendo assim, uma interdependência local-municipal.

As contradições entre o proposto e o implantado pelas gestões municipais ratificam que esse âmbito não assumiu o papel de porta de entrada da APS e a alternância de projetos políticos locais parece induzir rupturas nas diretrizes de governo. Isso pode afetar a organização das EqSF como contato preferencial dos sistemas municipais de saúde ${ }^{18}$.

Nas diretrizes municipais que tratavam da acessibilidade, a ênfase estava circunscrita à dimensão organizacional e com frágeis análises dos aspectos relativos ao âmbito geográfico, o que provoca uma avaliação superficial desse atributo no contexto estudado que possui grande extensão territorial e comunidades situadas na periferia e zonas rurais.

Ainda que tenham sido identificadas melhorias na acessibilidade das áreas rurais pela ampliação de cobertura de EqSF, em municípios com grande extensão territorial rural como o desse estudo, permanecem desafios de acessibilidade geográfica, seja grande distância entre comunidades e equipes de referência, seja indis- ponibilidade de transporte público regular nesses territórios.

A acessibilidade geográfica dos serviços de saúde diz respeito à organização de um dado território e relaciona questões como tempo, meio de deslocamento para serviços de saúde e situação econômica para arcar com o transporte, significando uma articulação entre acessibilidade geográfica e socioeconômica ${ }^{19}$.

A dimensão geográfica da acessibilidade na APS também não tem sido objeto dos estudos avaliativos sobre o tema no Brasil, que possui maior predomínio de pesquisas sobre acessibilidade organizacional. Isso porque os estudos compreendem que os obstáculos organizacionais no âmbito local e municipal possuem maiores impactos na acessibilidade dos serviços de APS, por tratarem diretamente de questões que envolvem uso do serviço, enquanto a dimensão geográfica seria um fator limitante menor, podendo influenciar, mas não, necessariamente, impedir a utilização do serviço ${ }^{8}$.

No âmbito internacional, a acessibilidade geográfica vem sendo investigada com mais frequência em países como Austrália e Escócia, principalmente, em regiões remotas e rurais que possuem mais dependência desse aspecto ${ }^{9,10}$. Destacam-se as desigualdades regionais de acesso geográfico aos serviços de APS, principalmente, em áreas remotas e rurais ${ }^{7}$. Em territórios da Austrália, Escócia e Eslovênia, foram evidenciadas limitações relativas à acessibilidade geográfica aos serviços de APS 9 .

No Brasil, tanto na percepção dos profissionais quanto dos usuários, as principais barreiras do acesso geográfico incluem a localização da unidade, as características locais, o transporte e a delimitação inadequada do território de abrangência $^{20}$. Tal percepção destaca a demarcação territorial das EqSF como um fator decisivo na implantação das unidades de saúde em locais que facilitem o deslocamento dos usuários da sua residência para o serviço.

As EqSF estudadas localizavam-se distante do CAE, sendo esse fato identificado como um aspecto limitante da acessibilidade geográfica, principalmente, considerando-se outras limitações físicas e financeiras para o deslocamento aos serviços especializados. Esses resultados apresentam-se coerentes com os estudos avaliativos da ESF que apontam problemas semelhantes relacionados ao acesso a serviços especializados ${ }^{21,22}$.

Há necessidade de avaliação das disparidades territoriais na saúde, especialmente a elaboração de territorialização eficaz, que leve em conside- 
ração a assistência prestada e a formulação de políticas públicas para além dos aspectos organizacionais, firmando-se em critérios geográficos e socioeconômicos territorialmente alinhados às necessidades da população ${ }^{16}$. Neste contexto, registram-se fatores que implicam na acessibilidade da APS: as necessidades de saúde e o modo de organização dos serviços ${ }^{23}$. As necessidades estão relacionadas, sobretudo, com a percepção de saúde dos usuários, que é um fator imediato na utilização do serviço ${ }^{24}$; enquanto a forma de organizar os serviços está relacionada ao reconhecimento das singularidades e particularidades de cada comunidade ${ }^{25}$.

Nessa pesquisa e em outros sistemas municipais de saúde do Brasil, usuários tendem a procurar a APS a partir de uma lógica assistencialista e de queixa-conduta. A busca pelo serviço das EqSF acontece, prioritariamente, pela ocorrência de algum problema de saúde e por considerar o atendimento mais rápido ${ }^{24}$. Dar-se também por ser necessária para o acesso a uma consulta com especialista ${ }^{17}$, bem como à facilidade para obter medicamentos, realizar os exames básicos e à referência para especialidades e exames especializados $^{26}$.

Outra semelhança deste estudo com demais cenários brasileiros refere-se à queixa dos usuários em relação à limitação dos horários de funcionamento das unidades de saúde à noite e finais de semana, que condicionava a busca de outros pontos de atenção de média e alta complexidade em casos de baixa urgência ${ }^{18}$. Iniciativas nacionais e internacionais implantaram estratégias de ampliação dos horários de atendimento em unidades de APS e encontraram resultados positivos na acessibilidade. Essa estratégia vem sendo adotada no Brasil, em Curitiba-PR, Mogi das Cruzes-SP e em Boa Vista-RR ${ }^{18}$, bem como em países como Reino Unido, Holanda e Estados Unidos7. Isso colaborou para redução do risco de fragmentação do cuidado, de superlotação dos serviços de emergência e auxiliou na redução de gastos com serviços de média e alta complexidade. Ressaltase que as dificuldades do acesso não se referem só aos horários de funcionamento das unidades, mas também aos processos de agendamento de consultas, de trabalho em equipe e de organização dos atendimentos à demanda espontânea ${ }^{27}$.

Em estudo prévio $^{8}$, a avaliação satisfatória do atendimento na UBS esteve vinculada à resolutividade dos problemas. A falta de solução para a demanda e o não atendimento à demanda espontânea, faz com que os usuários busquem serviços de emergências sejam até orientados pelos pro- fissionais das EqSF a procurarem outro serviço, gerando percepções negativas a respeito da APS. Desta forma, o problema relacionado à resolutividade abrange profissionais de saúde que trabalham na APS com baixa capacidade de tomar decisões, identificar problemas e propor soluções ${ }^{28}$.

A existência de demanda reprimida em algumas equipes foi realidade no estudo em questão. Muitos dos fatores explicativos relatados para chegar cedo e garantir o acesso a consultas de APS foram semelhantes com uma grande cidade do estado de São Paulo onde os usuários disputavam senhas de marcação ${ }^{15}$.

Nesse estudo, identificou-se inexistência de padronização no processo de marcação de consultas de APS. Em virtude das EqSF não se organizarem adequadamente para atender a oferta organizada e a demanda espontânea, a marcação de consultas é também relatada como fator limitante da acessibilidade em cidades como Ribeirão Preto-SP e Salvador-BA ${ }^{8,15}$. Ainda sobre o processo de marcação de consultas nas unidades de saúde, recomenda-se que as necessidades da população sejam as principais referências para estabelecimento da organização do funcionamento das EqSF, incluindo as formas de agendamento de consultas. Devem ser observadas possibilidades de adaptações para melhorar o acesso, seja agendamento por telefone, e-mail, entre outros ${ }^{29}$.

Os agentes eram elementos facilitadores do processo de marcação de consultas na percepção dos usuários entrevistados. Porém, essa prática de facilitação pelo ACS deve ser analisada com cautela, pois, não só reforça a importância desse trabalhador, como pode o usuário confundir essa ação como privilégio e a partir disso gerar frustrações para aqueles que não dispõem de $\mathrm{ACS}^{30}$.

$\mathrm{O}$ vazio de diretrizes municipais para marcação de consultas na APS denota que possibilidades de inovação nesse processo são desconcentradas para a gerência local das EqSF, estando dificilmente atreladas a mudanças induzidas pelo nível da gestão municipal. Um conjunto de barreiras está vinculado ao sistema de marcação de consultas, dentre elas: abertura de agenda em hora e dia determinado, estabelecimento de dias fixos para grupos populacionais e dificuldade de atendimento para demanda espontânea ${ }^{7}$. Tais fatores podem influenciar a procura por outros serviços de atenção à saúde, assim como corroborar para descrédito ao papel da APS como porta de entrada preferencial e ordenadora da rede de atenção à saúde ${ }^{15}$.

A baixa governabilidade das EqSF para ordenar o cuidado especializado reflete discrepâncias, 
de um lado, entre as necessidades de distribuição equitativa de vagas das consultas e exames para as equipes e, do outro, a insuficiência da oferta especializada nos sistemas municipais de saúde em regiões remotas como o Oeste baiano. Em sistemas municipais semelhantes ao do presente estudo, há concentração da oferta de AE nos prestadores privados, de modo que o quantitativo de consultas e exames para o SUS é determinado pelas vagas disponibilizadas na agenda dos prestadores locais e não pela gestão municipal ${ }^{7,12-14,20,31}$.

A marcação de consultas especializadas, rateadas em cotas para cada EqSF do município, não atendiam às necessidades locais dos serviços e implicava em baixo acesso dos usuários aos demais níveis da atenção. Esse ponto crítico restringia a capacidade da APS em cumprir sua função de porta de entrada, dada a dificuldade de referenciar usuários para os serviços especializados, já que a oferta dessas consultas depende, em grande parte, da disponibilidade de profissionais na rede, da quantidade de consultas disponibilizadas por eles no sistema de marcação e do quantitativo que foi pactuado com outros municípios da região de saúde. Esse fato foi semelhante aos municípios de Feira de Santana e Vitória da Conquista $^{31}$, bem como em grande cidade do estado de São Paulo ${ }^{15}$ e em município-sede da região Norte do estado Bahia ${ }^{32}$.

Os constrangimentos municipais sobre o nível local das EqSF indicam que a garantia da acessibilidade é multidimensional e sistêmica, dependendo, portanto, da ação de atores do âmbito primário, da gestão e de outros pontos de atenção que afetam a organização do sistema para garantir um fluxo de cuidado ordenado. $\mathrm{O}$ mau funcionamento do sistema de referência e contrarreferência foi um exemplo clássico de constrangimentos municipais da acessibilidade, assim como identificado na cidade de Salvador-BA ${ }^{23}$ e em município do Norte da $\mathrm{Bahia}^{32}$. Os fatores relacionados à alta demanda de usuários, ausência de comunicação e articulação com outros níveis de atenção à saúde dificultam a efetivação do sistema de referência e contrarreferência e influenciam na perpetuação de falhas no sistema de saúde e no cuidado integral da assistência ao usuário $^{33}$.

As práticas de acolhimento nas EqSF avaliadas mostraram-se limitadas ao contato da recepção. Essa era uma realidade que não correspondia àquilo que se encontra proposto nas diretrizes do acolhimento humanizado, que preveem ações que aproximem as práticas de saúde das reais necessidades dos usuários, estabelecendo conversa- ções, vínculo e servindo como ferramenta organizacional em toda a atividade de assistência, seja ela da demanda programada ou espontânea ${ }^{34}$. Sugere-se que o acolhimento deve ser entendido em três sentidos: como postura assumida pelos profissionais, como prática capaz de gerar procedimentos organizados e como princípio orientador de um projeto institucional ${ }^{28}$. Dessa forma, o acolhimento constituir-se-ia como diretriz operacional e que permitiria um atendimento humanizado, organização dos processos de trabalho em saúde, garantia de acesso universal e resolutividade.

Convém lembrar que o usuário faz as escolhas iniciais, a exemplo da opção por realização de consulta e local onde irá fazê-la, bem como, detém o poder de cumprir ou não as orientações e encaminhamentos. Logo, é importante considerar a subjetividade na avaliação da acessibilidade para compreendermos até onde a adequação da organização e oferta dos serviços se vincula às características dos usuários, considerando suas condições socioculturais, educacionais, econômicas, étnicas, de classe e gênero, seu estado de saúde, expectativas, necessidades e suas experiências.

Além de escutar a "voz ao usuário" é preciso provocar mudança nas relações de poder. Estimular a participação, não significa dar espaço para os usuários falarem, simplesmente, mas buscar a corresponsabilização com a disponibilidade para maior interlocução de saberes e construção de autonomia $^{35}$. A operacionalização do princípio da integralidade é possível através do reconhecimento dos usuários como sujeitos de direito. Contudo, as barreiras na acessibilidade das pessoas comprometem o cumprimento da integralidade e criam situações de injustiça social.

A acessibilidade constitui-se como atributo fundamental para o estabelecimento efetivo da APS como porta de entrada preferencial. Destarte, a APS torna-se lócus importante em relação ao combate às iniquidades sociais e na promoção da universalidade dos serviços, bem como da equidade e integralidade do cuidado. Nesse sentido, torna-se basilar identificar barreiras que dificultam a acessibilidade e que refletem em problemas nos modos de organização da assistência à saúde e nos modos de cuidar da população.

Os desafios para garantia da acessibilidade evidenciados neste estudo, demonstram que o cumprimento desse atributo está intimamente relacionado com determinantes municipais e locais das EqSF. Logo, para que haja o estabelecimento efetivo da APS como porta de entrada preferencial, faz-se necessária a valorização da 
acessibilidade e resolução dos seus fatores restritivos pela gestão.

As diferentes características das EqSF e territórios determinaram variações importantes na classificação geral da acessibilidade ao cuidado no cenário avaliado. Os contextos periféricos e rurais tendem a ser mais desafiantes para as EqSF superarem as barreiras geográficas da acessibilidade e os contextos urbanos as barreiras organizacionais.

Heterogeneidades no desempenho local da acessibilidade e identificadas nessa pesquisa traduzem uma importante variabilidade contextual do território e características do processo de trabalho das EqSF como fatores decisivos da coexistência de maiores ou menores obstáculos geográficos e organizacionais.

Destacam-se como contribuições deste estudo, sua amplitude analítica dos determinantes da gestão municipal e local da acessibilidade ao cuidado; a incorporação de múltiplas fontes a partir da ótica dos gestores, profissionais e usuários e de documentos municipais e das equipes, compondo um arcabouço avaliativo com maior profundidade; a avaliação da acessibilidade com inclusão de critérios geográficos e organizacionais, permitindo que seus resultados possam estabelecer conexões com a análise de outros sistemas municipais situados em regiões e com caracte- rísticas político-administrativas-organizacionais similares.

As principais limitações dessa pesquisa incluem a análise superficial dos determinantes geográficos, dadas justificativas já apresentadas nos resultados e a limitação para inclusão dos apoiadores da gestão da APS municipal, que inviabilizou maior profundidade na análise da organização do sistema, sob a ótica de outros atores que participam da gestão e monitoramento das EqSF.

São necessárias melhorias na organização do agendamento de consultas, acolhimento, na marcação de consultas especializadas, no sistema de referência e contrarreferência e no dimensionamento da distribuição espacial das unidades de saúde, sobretudo nas comunidades periféricas e rurais do município. Espera-se que os resultados deste estudo sirvam de base para subsidiar processos decisórios e de qualificação das EqSF. Recomendam-se futuras pesquisas que aprofundem a avaliação da acessibilidade na APS comparando modelos organizacionais. Questões como insucesso no sistema de referência e contrarreferência, acessibilidade geográfica e aspectos socioeconômicos da população e interseccionalidade de gênero, classe e etnia; assim como da acessibilidade política com foco no espaço de participação dos usuários devem ser considerados no intuito de ampliar o entendimento da acessibilidade na APS.

\section{Colaboradores}

MM Mendonça e IRS Aleluia participaram da concepção do estudo, coleta de dados e escrita do artigo. MLT Sousa e M Pereira contribuíram na escrita e revisão crítica do manuscrito. Todos os autores aprovaram a versão final do artigo. Declaramos para os devidos fins, que não possuímos qualquer tipo de conflito de interesse. 


\section{Referências}

1. Giovanella L, Mendonça MHM. Atenção Primária à Saúde. In: Giovanella L, Escorel S, Lobato LVC, Noronha JC, Carvalho AI, organizadores. Politicas e Sistemas de Saúde no Brasil. $2^{\text {a }}$ ed. Rio de Janeiro: Editora Fiocruz/Centro Brasileiro de Estudos de Saúde; 2013.

2. Lavras C. Atenção primária à saúde e a organização de redes regionais de atenção à saúde no Brasil. Saude Soc 2011; 20(4):867-874.

3. Batistão GT, Chaves MD, Gomes JO. Análise de dispositivos externos de acessibilidade nos serviços municipais de saúde: uma abordagem para deficientes físicos. Rev Bras Pesq Saude 2014; 16(4):32-38.

4. Camargos MCS, Gonzaga MR. Viver mais e melhor? Estimativas de expectativa de vida saudável para a população brasileira. Cad Saude Publica 2015; 31(7):1460-1472.

5. Brousselle A, Champagne F, Contandriopoulos AP, Hartz Z, organizadores. Avaliação: conceitos e métodos. Rio de Janeiro: Editora Fiocruz; 2011.

6. Donabedian A. An introduction to quality assurance in health care. New York: Oxford University Press; 2003.

7. Rocha SA, Bocchi SCM, Godoy MF. Acesso aos cuidados primários de saúde: revisão integrativa. Physis 2016; 26(1):87-111.

8. Gomide MFS, Pinto IC, Bulgarelli AF, Santos ALP, Serrano GMP. A satisfação do usuário com a atenção primária à saúde: uma análise do acesso e acolhimento. Interface (Botucatu) 2018; 22(65):387-398.

9. Figueira MCS, Silva WP, Silva EM. Acesso aos serviços da Atenção Primária em Saúde: revisão integrativa da literatura. Rev Bras Enferm 2018; 71(3):1178-1188.

10. Ford JA, Turley R, Porter T, Shakespeare T, Wong G, Jones AP, Steel N. Access to primary care for socio-economically disadvantaged older people in rural areas: A qualitative study. PloS One 2018; 13(3):e0193952.

11. Hlebec V. Evaluation of Access to Long-term Care Services for Old People Ageing in Place in Slovenia. $Z d r$ Varst 2018; 57(3):116-123.

12. Corrêa ACP, Ferreira F, Cruz GSP, Pedrosa ICF. Acesso a serviços de saúde: olhar de usuários de uma unidade de saúde da família. Rev Gaúcha Enferm 2011; 32(3):451-457.

13. Santos WJ, Giacomin KC, Firmo JOA. Avaliação da tecnologia das relações de cuidado nos serviços em saúde: percepção dos idosos inseridos na Estratégia Saúde da Família em Bambuí, Brasil. Cien Saude Colet 2014; 19(8):3441-3450.

14. Albuquerque MSV, Lyra TM, Farias SF, Mendes MFM, Martelli PJL. Acessibilidade aos serviços de saúde: uma análise a partir da Atenção Básica em Pernambuco. Saude Debate 2014; 38:182-194.

15. Cunha ABO, Silva LMV. Acessibilidade aos serviços de saúde em um município do Estado da Bahia, Brasil, em gestão plena do sistema. Cad Saude Publica 2010; 26(4):725-737.

16. Campos RTO, Ferrer AL, Gama CAP, Campos GWS, Trapé TL, Dantas DV. Avaliação da qualidade do acesso na atenção primária de uma grande cidade brasileira na perspectiva dos usuários. Saude Debate 2014; 38:252-264.
17. Pedraza DF, Nobre AMD, Albuquerque FJB, Menezes TN. Acessibilidade às Unidades Básicas de Saúde da Família na perspectiva de idosos. Cien Saude Colet 2018; 23(3):923-933.

18. Silva AN, Silva SA, Silva ARV, Araújo TME, Rebouças CBA, Nogueira LT. A avaliação da atenção primária a saúde na perspectiva da população masculina. Rev Bras Enferm 2018; 71(2):236-243.

19. Lima SAV, Silva MRF, Carvalho EMF, Pessoa EAC, Brito ESV, Braga JPR. Elementos que influenciam o acesso à atenção primária na perspectiva dos profissionais e dos usuários de uma rede de serviços de saúde do Recife. Physis 2015; 25(2):635-656.

20. Santinha G. Cuidados de saúde e território: um debate em torno de uma abordagem integrada. Saude Soc 2013; 22(3):815-829.

21. Silva LOL, Dias CA, Soares MM, Rodrigues SM. Acessibilidade ao Serviços de Saúde: Percepção de usuários e profissionais de saúde. Cogitare Enferm 2011; 16(4):654-660.

22. Silva LA, Casotti CA, Chaves SCL. A produção científica brasileira sobre a Estratégia Saúde da Família e a mudança no modelo de atenção. Cien Saude Colet 2013; 18(1):221-232.

23. Savassi LCM. Qualidade em serviços públicos: os desafios da atenção primária. Rev Bras Med Fam Comunidade 2012; 7(23):69-74.

24. Oliveira LSA, Almeida GN, Oliveira MAS, Gil GB, Alcione BOC, Medina MG, Pereira RAG. Acessibilidade a atenção básica em um distrito sanitário de Salvador. Cien Saude Colet 2012; 17(11):3047-3056.

25. Andersen RM. Revisiting the behavioral model and access to medical care: does it matter? J Health Soc Beha 1995; 36(1):1-10.

26. Santos WJ, Giacomin KC, Firmo JOA. Avaliação da tecnologia das relações de cuidado nos serviços em saúde: percepção dos idosos inseridos na Estratégia Saúde da Família em Bambuí, Brasil. Cien Saude Colet 2013; 19(8):3441-3450.

27. Mendes ACG, Miranda GMD, Figueiredo KEG, Duarte PO, Furtado BMASM. Acessibilidade aos serviços básicos de saúde: um caminho ainda a percorrer. Cien Saude Colet 2012 ; 17( 11 ): 2903-2912.

28. Pessoa BHS, Gouveia EAH, Correia IB. Funcionamento 24 horas para Unidades de Saúde da Família: uma solução para ampliação de acesso? Um ensaio sobre as "Upinhas" do Recife. Rev Bras Med Fam Comunidade 2017; 12(39):1.

29. Barbosa SP, Elizeu TS, Penna CMM. Ótica dos profissionais de saúde sobre o acesso à atenção primária à saúde. Cien Saude Colet 2013; 18(8):2347-2357.

30. Brasil. Ministério da Saúde (MS). Portaria no 2.436, de 21 de setembro de 2017. Aprova a Política Nacional de Atenção Básica, estabelecendo a revisão de diretrizes para a organização da Atenção Básica, no âmbito do Sistema Único de Saúde (SUS). Diário Oficial da União 2017; 24 set.

31. Cruz MJG, Hogla CM. Acessibilidade dos idosos na Rede Básica de Saúde. Rev Enferm UNISA 2009; 10(1):48-52. 
32. Almeida PFDE, Santos AM, Souza MKB. Atenção Primária à Saúde na coordenação do cuidado em Regiões de Saúde. Salvador: Edufba; 2015.

33. Aleluia IRS, Medina MG, Almeida PF, Vilasbôas AALQ. Coordenação do cuidado na atenção primária à saúde: estudo avaliativo em município sede de macrorregião do nordeste brasileiro. Cien Saude Colet 2017; 22(6):1845-1856.

34. Pontes APM, Cesso RGD, Oliveira DC, Gomes AMT. O princípio de universalidade do acesso aos serviços de saúde: o que pensam os usuários? Esc Anna Nery 2009; 13(3):500-507.

35. Sales IC. Os Desafios da Gestão Democrática. In: Anais IV Seminário Internacional de Representações Sociais, Subjetividade e Educação/VII Seminário Internacional sobre Profissionalização Docente. Recife; 2005. p. 16996-17009.

Artigo apresentado em 07/08/2020

Aprovado em 26/02/2021

Versão final apresentada em 28/02/2021

Editores-chefes: Romeu Gomes, Antônio Augusto Moura da Silva 